\title{
Promoting physical literacy and 24-hour movement in Hong Kong primary school children through a blended classroom-based intervention: protocol and baseline characteristics of the "Stand+Move" cluster-randomized controlled trial
}

\section{Ming Hui Li}

The Chinese University of Hong Kong https:// orcid.org/0000-0003-4116-3685

Raymond Kim Wai Sum ( $\nabla$ kwsum@cuhk.edu.hk)

The Chinese University of Hong Kong https://orcid.org/0000-0002-4051-9945

\section{Cindy Hui Ping Sit}

The Chinese University of Hong Kong

\section{Stephen Heung Sang Wong}

The Chinese University of Hong Kong

\section{Yun Kwok Wing}

The Chinese University of Hong Kong

\section{Research}

Keywords: physical literacy, sit-stand desk, physical activity, children, primary school, sleep, executive functions

Posted Date: April 14th, 2021

DOI: https://doi.org/10.21203/rs.3.rs-414376/v1

License: (c) (i) This work is licensed under a Creative Commons Attribution 4.0 International License. Read Full License 


\section{Abstract}

\section{Background}

As traditional classroom furniture contributes to children's predominant sitting during class, the study aimed to demonstrate the design and baseline characteristics of a three-armed intervention program, which targeting on enhancements in children's physical literacy, physical activity, sleep and executive function through constructing an active classroom environment in primary schools. The blended approach involves an organized physical activity participation during recess combining with the environmental changes during class in response to the 24-Hour Movement Guidelines in children and the emphasis on nurturing children's physical literacy in Hong Kong primary schools.

\section{Methods}

This blended "Stand + Move" intervention adopted a cluster-randomized controlled trial (RCT) design to investigate its effectiveness on health-related aspects and to compare: 1) a PA recess intervention group; 2) a blended (sit-stand desks and PA recess) experimental group; and 3) a control group. In total, 81 students were recruited and randomly assigned to the three groups for a period of 13 weeks (from March to September, 2019). The primary outcome is the children's physical literacy and physical activity. The secondary outcome is the children's sleep and executive functions.

Results

Among 81 participants enrolled at baseline, $40.7 \%$ of participants were boys and $59.3 \%$ were girls. The mean of age is 9.6 years [SD $=0.61$, range $9.0-12.0$ ]. There was no evidence of significant differences between trial arms at baseline concerning both the primary outcomes and secondary outcomes (all $P=$ 0.06-0.89). Of all, $22.4 \%$ met the recommended PA guideline, $36.8 \%$ met the sleep guideline, resulting in only $10.5 \%$ met both guidelines, and children's executive functions were evaluated in three aspects of inhibition, executive control, and planning. Over a half of the participants reported satisfactory in their perceived sleep quality.

\section{Discussion}

The designed intervention is regarded as an innovative strategy to reconstruct children's traditional classroom environment in Hong Kong. The baseline results suggest that the implementation of this study design was satisfactory. Therefore, it is expected to benefit the children's behavior-related health as well as the stakeholders in schools and families, and also provide the desired evidence to support policymaking reforms in teaching and learning strategies.

\section{Trial Registration}

ChiCTR, ChiCTR2000035038. Registered 29 July 2020 - Retrospectively registered, http://www.chictr.org.cn/hvshowproject.aspx?id=46038 


\section{Background}

Sedentary behavior (SB), defined as any low level energy expenditure, such as sitting, lying, reclining, or expending $<1.5$ metabolic equivalents (1), is an increasing issue in modern society. In fact, prolonged sitting has been linked to poorer physical health, psychosocial, and cognitive outcomes, irrespective of physical activity (PA) level (2). According to the Hong Kong Report Card 2018, over $90 \%$ of Chinese school-aged children and youth insufficiently participate in PA (2). The grade achieved for children's SB was $\mathrm{C}$-, indicating that slightly over half $(52 \%)$ of primary school children, mean age 7.6 years, spent $<2 \mathrm{~h}$ on screen time (2). For children attending primary school, sitting is a common behavior as they are predominantly seated in a classroom for the duration of their day, excluding physical education (PE) classes and breaks. Previous studies have provided evidence that Hong Kong children spend up to $32.3 \%$ of their waking time sitting, with their reported sitting time approximately $4.9 \mathrm{~h} /$ day (3), while in some studies, the reported sitting time is up to $10 \mathrm{~h} /$ day (4). High levels of SB have been negatively associated with cardio-metabolic health risk markers, such as obesity, blood pressure, cholesterol, and insulin, in children $(5,6)$. More importantly, SB can be tracked throughout childhood and into adolescence and adulthood (7). Therefore, disrupting prolonged sitting habits, and fostering healthy alternatives during childhood, is paramount for promoting a healthy lifestyle in adulthood.

Positive associations have been found between PA and classroom behavior and learning $(8,9)$. Public health authorities have recommended that primary schools assist children to meet the PA guideline of 60 min moderate-to-vigorous PA (MVPA), through a comprehensive approach incorporating both activities during class, recess, and before-and-after school (10). The concept of physical literacy (PL) is also emphasized as an important ideology to encourage each child to move with "the motivation, confidence, physical competence, knowledge, and understanding to value and take responsibility for engagement in physical activities for life" (11), a concept first introduced to PE teachers in Hong Kong through Continuing Professional Development (CPD) provided by the Education Bureau (12). Recently, a holistic 24-Hour Movement Guideline for Children and Youth was proposed in response to global perspectives on children's PL development. This emphasized PA, SB, and sleep as three co-developmental movement behaviors relating to a full range of movement within a $24 \mathrm{~h}$ period (13). This guideline provides specific daily recommendations, including MVPA ( $\geq 60 \mathrm{~min} /$ day), screen time ( $\leq 2 \mathrm{~h} /$ day), and sleep (9$11 \mathrm{~h} /$ night, for children aged 5-13 years, and 8-10 h/night for adolescents aged $14-17$ years). A comprehensive approach involving the PL concept, which incorporates $\mathrm{PE}$, recess, and PA opportunities in both the classroom and before-and-after school, is helpful for children to meet the movement guideline within the 24-hour period (10). As such, interventions should target on a blended design to improve primary schoolchildren's physical activity and physical literacy and enable them to reduce prolonged sitting for an active and healthy lifestyle throughout their lifespans.

\section{Fostering children's physical literacy and physical activity in the school setting}


The conceptual framework of Cairney et al (2019) suggests that the development of children's PL, early in life, will influence their subsequent participation in PA and related outcomes across the life course (14). There is also evidence that among school-aged children, $\mathrm{PL}$ is the foundational component contributing to various health indicators, with PA playing a mediating role. Therefore, fostering PL and PA in schoolaged children is of paramount need. PA interventions must align with relevant systems (e.g., health, education, local government, etc.), and have scope to be feasible, cost-effective, and policy-relevant to achieve maximum impact and effectiveness in school-aged children (15). In this case, interventions focusing on school environments could be one of the feasible, convenient and cost-effective measures for children to develop their PA and reduce prolonged sitting time $(16,17)$. To foster children's habit of maintaining engagement in PA and reduction of sedentary time, strategies that will support children to break up prolonged sitting time and trigger more standing and moving, seem feasibly beneficial for their health. Furthermore, children's sitting behaviors tend to occur habitually and automatically, without conscious thought. Their habitual behaviors may have also been formed at an early age in school. In this case, favorable contexts or environments that trigger children's participation in PA are essential targets for conducting the interventions (15). For example, automatic sitting behavior is pervasive when using chairs and seated height tables. Children are also under the control of their teachers/parents or other adults who are responsible for their behavior and whose instructions may often require them to sit still in class or in front of the television. It is important to adopt novel strategies and assess effective ways to reduce children's SB.

In addition to breaking up prolonged sitting in class, incorporating short bouts of activity throughout the school day could help children accumulate the required amount of PA and foster their PL. These measures could be provided outside traditional periods-break times and physical education (PE) classes -for children to be active. Due to budgetary constraints and growing pressure on administrators and teachers to increase academic achievement scores, opportunities for PA are being reduced or eliminated. An effective environmental approach to increase PA has been to incorporate activity breaks into the primary school schedule (18). PA breaks comprise a low-cost, teacher-directed intervention that only needs support in the form of attractive resources, such as student posters, teacher notes, or a music CD (19). The intervention was reported to result in a significant increase in daily steps and children undertaking more PA during school hours than the controls (19). It is hoped that the current study's

blended "Stand + Move" design will contribute to breaking up prolonged sitting due to contextual constraints from the traditional classroom as well as increasing PA engagement during school time.

\section{Children's 24-hour movement behavior and executive functions (EFs)}

Sedentary behaviors, including screen time together with PA and sleep, have recently been integrated as "movement behaviors." Researchers suggest that complying with adequate levels of these behaviors may optimize children's health and development over a 24-h period. Previous studies provide evidence that in early childhood, PA has positive associations with cardiometabolic, physical, and psychosocial development, as well as in improving children's motor and cognitive development (20), especially EFs. It 
comprises a series of higher-order cognitive processes that develop rapidly during the early years (21), and routinely includes aspects of memory, inhibitory control, and cognitive flexibility to organize or coordinate behaviors when performing complex tasks (22). Meanwhile, individual evidence has proved that sleep (23), PA (24), and screen-based SB (25) are all associated with psychological health and early $\mathrm{EFs}$, but the influences of these behaviors' integration is unclear as few studies have investigated the associations of combinations of movement behaviors with cognition in early childhood. However, only one observational study has provided evidence that there are positive associations between cognition with each additional recommendation met (26). Given that movement behaviors are interrelated, health benefits may be optimized when all components of movement behavior are considered (27).

The aggregation of movement behaviors should also consider a combination of breaking up prolonged sitting, by specially focusing on reducing typically observed SB among primary school children before the transition into adolescence. A study that explored breaking up of prolonged sitting found that it often contributed to less attention in class (28). Previous literature reflects a lack of clarity in terms of the relationship between SB or PA and the different aspects of cognitive functions. Therefore, it is necessary to include EFs as one of the measures to evaluate the effectiveness of the active classroom intervention. These aspects of EF tasks include not merely remembering important details and updating rules, but refer to inhibiting movements, shifting flexibility between different situations, and planning and acting in anticipation of an event, which would lead to better academic achievement (29).

As school-aged children, during most lessons, are expected to sit in the traditional classroom setting, outside their limited PE lessons, it seems impossible for them to meet the aforementioned guideline within each 24-h period (30). Therefore, in order to reduce children's sitting time and promote PA, practical strategies are needed to reconstruct the classroom environment so that children may develop their PL and reduce their engagement in SB (31). The "Stand + Move" program, which can be feasibly achieved by children to develop their PL in the school setting, also reducing their engagement in $\mathrm{SB}$, is one such strategy, combining sit-stand desks with PA breaks. Unlike traditional classroom desks, sit-stand desks are height adjustable, enabling the child to alternate between sitting and standing postures. As previous research has reported a significant increase in SB in children aged 11 years and older, relative to younger age groups (32), it is necessary to reduce typically observed SB among primary school children before transition into adolescence.

In Hong Kong, to the best of our knowledge, interventional studies targeting height-adjustable tables and PA breaks have not been conducted in a school setting. As such, this pioneer study incorporates sit-stand desks and PA breaks as the active strategy behind not only reducing students' sitting time and increasing their PA engagement, but also investigating the benefits of this intervention on children's PL, sleep patterns, and executive functioning, thus promoting an all-round healthy lifestyle for students in Hong Kong primary schools. It is hypothesized that the blended "Stand + Move" group, compared with the single "Move" group and the control group, increases mostly in: 1) their self-perceived PL and actual PL; 2) objectively-measured PA, SB and sit-stand transitions; 3) executive function in the domains of inhibition, executive control and planning; 4) sleep patterns including sleep duration, sleep hygiene, sleep 
disturbance, and daytime sleepiness. Both the individual level (the self-reported and objectively-measured outcomes) and the cluster level (compare differences between groups) are included. As such, in this paper we demonstrate the study protocol and baseline characteristics of the intervention program utilizing a cluster randomized controlled trial (RCT) design, and report the baseline characteristics of the trial population according to the CONSORT Statement for cluster randomized-controlled trials (Supplementary file 1) (33).

\section{Methods}

\section{Study design}

The "Stand + Move" intervention was designed as a three-arm RCT study to evaluate the effectiveness of the blended "Stand + Move" intervention program. Outcome data are collected at baseline, postintervention and 3-month follow-up in self-perceptions and objective measures of all variables. Baseline data collection was conducted before the intervention began in the two intervention arms. The postintervention measure for all the clusters will be performed successively 13-week after the completion of the intervention aiming to reduce children's SB. The follow-up measurement in all clusters will be performed 3 months after the post-intervention measurement. Baseline data collection took place between September and November 2020. The measurements at post-intervention and follow-up will take place in 2021.

The present study focused on approximately 9-year-old $4^{\text {th }}$ grade students in Hong Kong and the ethical approval was obtained from Survey and Behavioral Research Ethics of the Chinese University of Hong Kong. Clusters were regarded as the classes recruited from one public school located in New Territories, Hong Kong. Following the initial recruitment process, baseline assessments were conducted at participating schools. Prior to their participation, written informed consent were provided by the parents/guardians of all children. All the personal information about potential and enrolled participants were kept confidentially before, during, and after the trial, and only the project members have access to the final trial dataset under the aim of the data analysis. Figure 1 shows a flow chart of this study. The clusters of participants were randomly assigned (using Google random number generator) into one of the following three groups: a single PA break intervention group (PA), a blended intervention group (combining sit-stand desks and PA breaks; SSPA), or a control group (CG). A blended intervention design was adopted, as it was considered an innovative design for disrupting prolonged sitting and improving engagement in PA, a pragmatic measure of school-aged children's PL (34). In addition, this intervention was regarded as a key facilitator of the Comprehensive School PA Program (CSPAP), a necessary approach for fostering school-aged children to develop their PL, considering that schools play a critical role in reshaping both social and physical environments, as well as providing information, tools, and practical strategies to help students adopt healthy lifestyles (10). Primary outcomes included children's PA level, including MVPA and sedentary time, and PL level (self-perceptions and actual PL) (35). Secondary outcomes consisted of children's personal status, such as body weight and height, their performances in executive functions, academic achievement, and changes in sleep patterns and duration. 
As the current study was led by the teachers who are required to implement the interventions in schools, they were blinded to group allocation. Students were not informed about the research aims and related information, and was therefore blinded to group allocation. Research assistants who are responsible for data collection were not blinded to group allocation.

\section{Participants and recruitment}

As previous research reported a significant increase in SB in children aged 11 years and older (32) and reduced the typically observed SB before transition into adolescence, this study recruited students from fourth grade of Hong Kong primary schools (9-10-year-old). G-power software was used to calculate sample size with an alpha of 0.05 , power of $80 \%$, and effect size (r) of $0.3(36)$. Since a full desk allocation system (a sit-stand desk for every child) guaranteed the optimal health benefits for the children as they could have maximum exposure to the desks, only one school was approached and recruited, with a total number of 81 participants, who was randomly assigned to either of the three conditions with a 1:1:1 ratio (Figure 1). Children were excluded if they: (a) not $4^{\text {th }}$ grade; (b) had a disability that prevented periods of standing; or (c) had an injury or illness that limited performing normal daily tasks.

Consent forms were distributed to all the $4^{\text {th }}$ grade students' parents by the school teachers. A briefing session on the aims and procedures of the intervention was held for all the teachers and parents, who agreed to participate in the entire intervention process. Participants' personal data was kept confidential, and was informed that they can voluntarily withdraw from the project at any time, without prejudice. Upon reaching into an agreement with the principals, teachers, and parents, the trained appraisers started data collection after obtaining informed consent.

\section{Baseline measures}

\section{Primary outcomes}

\section{Physical literacy - actual level}

Body mass index (BMI, $\mathrm{kg} / \mathrm{m} 2)$ was calculated from the measured weight (nearest $0.1 \mathrm{~kg}$ ) and height (nearest $0.1 \mathrm{~cm}$ ). PL was assessed using the Chinese version of the Canadian Assessment of PL, second edition (CAPL-2, Chinese) (37), a comprehensive protocol that can accurately and reliably assess a broad spectrum of skills and abilities that contribute to and characterize the PL level of a participating child (38). It comprised four domains: Daily Behavior; Physical Competence; Knowledge and Understanding; and Motivation and Confidence. The total achievable score for this assessment was 100 points. Daily Behavior comprised two parts: objectively-measured step counts and self-reported moderate-to-vigorous physical activity (MVPA) (i.e., the number of days in a week that children engaged in activities that made them breathe harder and their heart beat faster). The total achievable score for this assessment was 30 points. Physical Competence consisted of three parts: i) FitnessGram 15 m/20 m Progressive Aerobic Cardiovascular Endurance Run (PACER) (39) for evaluating aerobic fitness; ii) Plank Assessment of Torso Strength (40) for testing musculoskeletal endurance related to back health, the ability to stabilize the 
body, and the functioning of both the upper and lower limbs; and iii) the Canadian Agility and Movement Skill Assessment (CAMSA) for assessing motor competence (41). The total achievable score for this assessment was 30 points. The Knowledge and Understanding domain assessed a child's PL-related knowledge, with five questions equaling a total of 10 points. The Motivation and Confidence domain evaluated a child's confidence in his/her ability to be physically active, as well as his/her motivation to participate in PA. A revised version of the "What's most like me," the Children's Self-perceptions of Adequacy in and Predilection for Physical Activity (CSAPPA) questionnaire (42), was adopted for assessing this domain with a total of 30 points, which was adequately evaluated for model fit after revisiting the concept of PL (43). The whole CAPL-2 (Chinese) model was reported a good fit with construct validity: chi-square $\left(\chi^{2}=70.16, d f=43, p<0.05\right), \mathrm{RMSEA}=0.04,90 \% \mathrm{Cl}(0.024-0.062), \mathrm{CFI}=$ $0.94, \mathrm{TLI}=0.90$, to be adopted to evaluate children's PL.

\section{Perceived physical literacy}

PL perceptions was assessed using the adolescent version of the Perceived Physical Literacy Instrument (PPLI), a nine-item questionnaire (44). Each response was rated on a 5-point Likert scale ranging from strongly disagree to strongly agree. Adapted from a previous version constructed by PE teachers (45), the current questionnaire's validity was confirmed through confirmatory factor analysis (CFA): chi-square $\left(\chi^{2}\right.$ $=321.54, d f=24, p<0.05), \mathrm{CFI}=0.95, \mathrm{RMSEA}=0.08$, and standardized root mean square residual $(S R M R)=0.04$. Furthermore, the questionnaire showed acceptable reliability, with a values ranging from 0.68 to 0.76 .

\section{Physical activity}

Children's PA was monitored by two different types of activity devices, namely ActiGraph GT3X+ (46) and activPAL ${ }^{\text {TM }}$ accelerometers (PAL Technologies, Glasgow, United Kingdom). The ActiGraph monitors were worn on the children's waists for seven consecutive days. Data was collected in $15 \mathrm{~s}$ epochs to account for children's natural activity levels, which generally occurred in short bouts (47) as it was shown to present the most acceptable classification accuracy for accelerometer use among children. Evenson cutpoints (MVPA $\geq 2296$ counts min-1) was applied to intensity levels. The ActiGraph monitors had to be worn for at least $8 \mathrm{~h}$ /day for a minimum of 4 days, with at least one valid weekend included (48). The accelerometers could be removed only during water activities, such as showering or swimming, and the participants had to fill in the information provided in the log sheets. While the activPAL monitors were worn on the midline of children's right thighs and it could be used to detect limb positions, such as sitting/lying, standing, and stepping (49). Similar to ActiGraph, a continuous 7-day-wearing waterproof protocol was adhered to ensure the monitoring of children's PA and SB during a whole $24 \mathrm{~h}$, and the activPAL data was divided into 15-s periods with meeting a minimum requirement of 3 valid weekdays and 1 valid weekend day $(50,51)$. The activPAL data was summarized into time spent sitting/lying, standing and stepping.

\section{Secondary outcomes}




\section{Executive functions}

\section{Inhibitory control}

EFs, including inhibition, executive control, and planning were assessed by three computer-based tasks, all of which was performed using the Inquisit 5 platform. Participants were required to perform three tasks, one by one, in a quiet room under the supervision of an instructor who was trained prior to the testing. Inhibition control was examined using a modified version of the Eriksen flanker task (52). This task consisted of five arrows on a screen, and participants were asked to determine the direction of the target arrow in the middle. The arrows pointing to the left " $<$ " and right " $>$ " directions required a right and left keyboard button response, respectively. The two flanker arrows on each side of the target arrow worked as the distractors and would appear as either congruent trial ">>>>>" or congruent trial ">><>>." Each stimulus was shown for 120 milliseconds (ms), and the participants were required to respond within 200 to $1750 \mathrm{~ms}$ from the onset of the arrows, for a valid response. This task contained 4 practice trials and 20 test trials, with an equal number of congruent and incongruent trials occurring in a random order. The outcomes included two domains: accuracy (percentage of correct responses) and reaction time (number of ms for correct responses).

\section{Executive control}

Executive control was measured with the classical version of the Wisconsin Card Sorting Test (WCST) with the standard number of 128 cards (53). This task consisted of 4 key cards and 128 response cards. Participants were instructed to sort the response cards, shown at the bottom of the screen, according to the characteristics of the key cards presented on the screen's upper side, comprising the following categories: colors (red, green, yellow, and blue); forms (triangle, star, cross, and circle), and numbers (1-4). The instructor was permitted to provide instructions relating to the categories either prior to or during the task, while feedback on "correct" or "incorrect," was presented after each selection. It took each participant approximately 20 mins to complete the task. Both total and perseverative errors were recorded as executive control variables, since an increase in any of these variables suggested executive control impairment (54). While the calculation of total errors were based on the number of times participants match a card incorrectly, perseverative errors were based on the participants' continuing to follow the previous error rule.

\section{Executive planning}

The Tower of London Task, a widely administered neuropsychological assessment were used for measuring the planning aspects of EFs (55). The task consisting of a practice trial and 12 test trials required the participants to move beans to solve problems. When presented with a graph on the screen showing three vertical pegs with graded heights and each holding beans (either 3,2 , or 1 ), the participants had to move the beans so as to be identical to the goal graph, without violating the rules (56). It took each participant approximately 20 mins to complete the task. Both the total correct and total move scores were 
derived for analysis, given that these variables were found to be influenced by aerobic and resistance exercises (56).

\section{Children's Sleep}

The Children's Report of Sleep Patterns (CRSP) questionnaire, containing 60 items pertaining to three modules-Sleep Patterns, Sleep Hygiene Index, and Sleep Disturbances Scale-were used to measure different aspects of sleep among children aged 8 to 12 years (57). The questionnaire's psychometric properties had been tested in a population of 456 children using a multi-method, multi-reporter approach, and are reported to have good reliability and validity. This assessment was administered in the current study. In addition, considering the negative impact of sleep loss on grades and overall daytime functioning in children, daytime sleepiness was assessed using the Pediatric Daytime Sleepiness Scale (PDSS) (58). This was a parent-reported instrument consisting of 8 items, having $>0.40$ acceptable factor loadings. Internal consistency of the total 8 -item scale (factor 1 , PDSS) was a $=0.81 / 0.80$ for the splithalf samples.

\section{Data collection procedures}

During the first stage of data collection, participants were required to complete both the CAMSA and Plank during the PE class. The participants were divided into two groups, with one or two appraisers per group, and rotated around the stations (one test per station) till the assessment was completed. Prior to CAMSA, they watched two test presentations performed by an appraiser. During the first presentation, the appraiser covered the entire course at a slow pace with detailed verbal descriptions of each skill. While the second presentation was at full speed, the appraiser maintained skill accuracy. Participants were required to practice twice, at full speed, while maintaining their skill accuracy. Each participant's highest combined time and skill score was recorded as his/her final grade. In the Plank test, the participants watch the demonstrations first. Thereafter, stopwatches were used to record when each participant achieved the right posture. One warning was announced when participants' positions were too low/high or they were unable to maintain their position. The recording was stopped when participants shifted their positions a second time.

During the second stage of data collection, participants' aerobic fitness was monitored based on their participation in the PACER $15 \mathrm{~m} / 20 \mathrm{~m}$ shuttle run during their scheduled PE classes. Due to limited space, all participants ran from one marker to another set $15 \mathrm{~m}$ apart, while keeping pace with a prerecorded Cantonese cadence. The total number of laps achieved by each participant was recorded, and then converted to the standardized $20 \mathrm{~m}$ PACER score using the FitnessGram PACER Conversion Chart (39). Lastly, ActiGraph and activPAL accelerometers were distributed to the participants to monitor their step counts, PA and SB for seven consecutive days.

Height and weight measurements, questionnaire completion, and participation in the cognitive tests all took place during music, science, or other classes, and not during the PE classes. Two participants visited 
the quiet experimental room at a time to complete the aforementioned measurements under the instruction of two trained helpers.

\section{Process evaluation}

Process evaluation (often called formative evaluation) aims to improve a policy or program as it was being implemented (59). With regard to feasibility, evaluation should check whether and to what degree the implementation is accomplished, such as context, reach, fidelity, acceptability implementation, impact, acceptability, and sustainability over time through a pragmatic design (60). In the current study, a qualitative methodology will be adopted to examine the perceptions and experiences of key stakeholders in the intervention schools, as the interpretivist paradigm illustrates that human action and interaction, in the school setting, is experienced subjectively evaluated through individual meaning making (61). Key stakeholders, such as teachers and students, are inherently associated with the effectiveness of blended "Stand + Move" intervention. This method will include semi-structured interviews with the teachers involved in the intervention group and focus groups with randomly selected students after measurements to avoid its influence on the results. Besides, classroom observations will also be conducted during the intervention period by research team members, who recorded field notes based on these observations (62), including positive or negative responses to the enforced sit-stand desks utilized during class, the children's attitude towards sit-stand desks during the class and PA breaks during the recess, and sitting and standing behavior immediately after the enforced sit-stand desks implementation.

\section{Results}

All data was imported into SPSS version 23 for analysis. An a level of 0.05 was used for all statistical tests. Prior to analyzing data, several methods were adopted to replace any missing values in the outcome variables. The missing values in the Physical Competence and the Daily Behavior domains of PL were calculated according to the fraction provided in the CAPL-2 (Chinese) manual. A score could still be calculated when a maximum of one protocol was completely missed (43). For measuring PA, an individual information-centered approach was adopted for substituting missing data points (63). This had been demonstrated as an effective method, and superior to the group information-centered methods for handling missing accelerometer data when data was collected for 7 days. A total of 76 students $(59.2 \%$ girls, Mage $=9.6$ years $[S D=0.61$, range $9.0-12.0])$ were evaluated after completing the missing data. Daytime sleepiness data was not available for 8 participants (SSPA $=3 ; P A=1 ; C G=4$ ) as the questionnaires were not returned from their parents.

Table 1 shows the baseline demographic characteristics of all the participants. The majority (69.4\%) of the participants' parents obtained at least college or university level of education, and the family house type and the income status reflected the socio-economic demographic status of the participants in three groups. Descriptive statistics was expressed as frequencies, ratios, and means with standard deviations. Shapiro-Wilk and Levene's tests were used to check the normality and homogeneity of data. Multivariate analysis of variance (MANOVA) test was used to assess between group comparisons at baseline. Table 2 
displays the baseline descriptive statistics of primary outcomes with group differences. During the wear of activPALs, nearly half of the participants reported allergic symptoms for the waterproof hypoallergenic tape (3M Tegaderm ${ }^{\mathrm{TM}}$; $3 \mathrm{M}$ Health Care $^{\mathrm{TM}}$, St. Paul, MN; $10 \mathrm{~cm} \times 10 \mathrm{~cm}$ ), leaving only 41 valid data for analysis (SSPA $=22 ; P A=7 ; C G=11)$. There was no significant difference between groups in all the primary outcomes $(P=0.055-0.808)$, except a significant group difference was observed in the standing time measured by activPAL between PA group and CG $(p=0.017)$. The baseline descriptive statistics of secondary outcomes with group differences were shown in Table 3. No significant group differences were found in all the secondary outcomes $(P=0.133-0.886)$. Several aspects of sleep were particularly selected and shown in Table 4, as these could reflect children's perceived sleep quality and habits. Over a half of the participants in each group has reported a satisfactory sleep patterns in duration (SSPA = $60.9 \%$; $P A=80 \%$; $C G=75 \%$ ), and very few children reported their perception for sleep quality (SSPA $=4.3 \%$; $\mathrm{PA}=4.0 \% ; \mathrm{CG}=3.6 \%$ ) are poor. According to the 24-Hour Movement Guidelines in Children and Youth (64), $22.4 \%$ of the participants met the recommended PA guideline, $36.8 \%$ met the sleep guideline, resulting in only $10.5 \%$ met both guidelines. 
Table 1

Baseline demographic characteristics of the study participants

\begin{tabular}{|c|c|c|c|}
\hline & $\begin{array}{l}\text { SSPA } \\
(n=23)\end{array}$ & $\begin{array}{l}P A \\
(n=25)\end{array}$ & $\begin{array}{l}\text { CG } \\
(n=28)\end{array}$ \\
\hline \multicolumn{4}{|l|}{ Demographic } \\
\hline Gender (Female) & $15(60 \%)$ & $16(57.1 \%)$ & $17(60.7 \%)$ \\
\hline Age, mean (SD) & $9.7(0.7)$ & $9.6(0.6)$ & $9.6(0.6)$ \\
\hline Height, mean (SD) & $136.3(6.6)$ & $134.8(8.7)$ & $136.3(4.3)$ \\
\hline Weight, mean (SD) & $31.6(7.5)$ & $32.3(9.0)$ & $31.6(6.3)$ \\
\hline BMI (kg/m2), mean (SD) & $16.8(3.0)$ & $17.3(3.1)$ & $16.9(2.8)$ \\
\hline \multicolumn{4}{|c|}{ Socio-demographic characteristics } \\
\hline \multicolumn{4}{|c|}{ Parent's highest level of education completed } \\
\hline Secondary school (\%) & 31.8 & 41.7 & 19.2 \\
\hline College (\%) & 31.8 & 16.7 & 26.9 \\
\hline University (\%) & 34.8 & 33.3 & 50.0 \\
\hline Postgraduate (\%) & 0 & 8.3 & 3.8 \\
\hline \multicolumn{4}{|l|}{ House type } \\
\hline Public housing estates (\%) & 21.1 & 0 & 26.3 \\
\hline Private housing estates (\%) & 57.9 & 89.5 & 68.4 \\
\hline Single House (\%) & 5.3 & 10.5 & 0 \\
\hline Others (\%) & 15.8 & 0 & 5.3 \\
\hline \multicolumn{4}{|l|}{ Family monthly income } \\
\hline Less than $40,000(\%)$ & 40.9 & 21.7 & 29.2 \\
\hline From 40,000 to $70,000(\%)$ & 36.4 & 47.9 & 45.8 \\
\hline Greater than 70,000 (\%) & 22.7 & 30.4 & 25 \\
\hline
\end{tabular}


Table 2

Baseline characteristics of primary outcomes and group comparison

$\begin{array}{llll}\text { SSPA } & \text { PA } & \text { CG } & p= \\ (n=23) & (n=25) & (n=28) & \end{array}$

Physical Literacy - Actual level

Motivation \& Confidence

$22.8 \pm 4.8$

$21.4 \pm 5.0$

$22.0 \pm 4.8$

0.619

Physical Competence

$14.7 \pm 5.7$

$13.3 \pm 3.5$

$14.6 \pm 4.0$

0.351

Knowledge \&

$4.5 \pm 1.9$

$4.6 \pm 1.3$

$4.0 \pm 1.8$

0.254

Understanding

Daily Behavior

$10.5 \pm 3.5$

$9.4 \pm 3.2$

$10.8 \pm 3.6$

0.330

Perceived Physical Literacy

SS_PPL

$11.3 \pm 2.5$

$10.9 \pm 1.9$

$11.0 \pm 1.6$

0.760

SE_PPL

$11.4 \pm 2.4$

$11.1 \pm 1.4$

$11.1 \pm 1.9$

0.808

KU_PPL

$12.6 \pm 2.5$

$12.1 \pm 1.6$

$12.5 \pm 1.5$

0.658

Physical Activity - Actigraph

Total wear time (mins)

$4680.3 \pm$

1412.2

$4387.8 \pm$

1672.0

$3844.7 \pm 1678.3$

0.191

Sedentary (\%)

$67.3 \pm 7.4$

$66.8 \pm 5.1$

$68.3 \pm 7.2$

0.661

LPA (\%)

$28.0 \pm 5.2$

$28.0 \pm 4.2$

$26.8 \pm 5.8$

0.667

MVPA (\%)

$5.1 \pm 2.0$

$5.2 \pm 1.8$

$5.0 \pm 2.0$

0.622

Physical Activity - activPAL

Sitting time ${ }^{a}$

$1183.1 \pm 81.0$

$1212.6 \pm 82.2$

$1184.3 \pm 85.1$

0.700

Standing time ${ }^{\text {a }}$

$220.8 \pm 44.4$

$190.5 \pm 49.5$

$244.7 \pm 42.7$

$0.055^{\#}$

Stepping time ${ }^{a}$

$125.7 \pm 21.3$

$105.8 \pm 28.2$

$114.0 \pm 23.3$

0.111

Sit-to-stand transitions ${ }^{\text {a }}$

$107.8 \pm 21.0$

$98.3 \pm 31.5$

$103.1 \pm 20.1$

0.612

$\# p<0.10 ;{ }^{a} \mathrm{n}=41$;

Abbreviations: SS_PPL, SE_PPL, and KU_PPL are the attributes of perceived physical literacy; LPA = light physical activity; MVPA = moderate-to-vigorous physical activity; SSPA = Sit-stand desks and PA blended group; PA = single PA group; $C G$ = Control Group 
Table 3

Baseline characteristics of secondary outcomes and group comparison $(M, S D)$

\begin{tabular}{|c|c|c|c|c|}
\hline & $\begin{array}{l}\text { SSPA } \\
(n=23)\end{array}$ & $\begin{array}{l}P A \\
(n=25)\end{array}$ & $\begin{array}{l}C G \\
(n=28)\end{array}$ & $p=$ \\
\hline \multicolumn{5}{|l|}{ Sleep } \\
\hline Sleep time (h) & $8.2 \pm 0.7$ & $8.3 \pm 1.2$ & $8.4 \pm 0.8$ & 0.498 \\
\hline Sleep hygiene & $32.6 \pm 5.1$ & $31.0 \pm 7.6$ & $32.5 \pm 7.8$ & 0.680 \\
\hline Sleep disturbance & $27.6 \pm 5.8$ & $26.0 \pm 7.8$ & $28.0 \pm 10.8$ & 0.667 \\
\hline Daytime sleepiness ${ }^{\text {a }}$ & $13.6 \pm 5.6$ & $14.6 \pm 5.0$ & $13.5 \pm 5.8$ & 0.786 \\
\hline \multicolumn{5}{|l|}{ Inhibition } \\
\hline Accuracy - congruent (\%) & $99.6 \pm 2.0$ & $97.8 \pm 4.2$ & $98.6 \pm 4.5$ & 0.355 \\
\hline Accuracy - incongruent (\%) & $94.2 \pm 18.6$ & $90.0 \pm 21.3$ & $95.0 \pm 12.0$ & 0.580 \\
\hline RT - congruent (ms) & $626.6 \pm 167.4$ & $698.8 \pm 203.7$ & $642.2 \pm 174.1$ & 0.256 \\
\hline RT - incongruent (ms) & $795.6 \pm 353.2$ & $774.7 \pm 338.6$ & $732.7 \pm 239.0$ & 0.769 \\
\hline \multicolumn{5}{|l|}{ Executive control } \\
\hline Total errors ${ }^{\wedge}$ & $43.3 \pm 19.2$ & $51.4 \pm 15.2$ & $52.9 \pm 18.0$ & 0.138 \\
\hline Perseverative errors ${ }^{\wedge}$ & $7.5 \pm 2.4$ & $6.9 \pm 4.4$ & $8.3 \pm 7.2$ & 0.591 \\
\hline \multicolumn{5}{|l|}{ Planning } \\
\hline Total correct & $74.0 \pm 12.7$ & $73.3 \pm 11.4$ & $71.6 \pm 14.4$ & 0.886 \\
\hline Total move scores & $29.0 \pm 3.2$ & $29.4 \pm 2.5$ & $27.8 \pm 3.8$ & 0.133 \\
\hline \multicolumn{5}{|l|}{ a $=68$} \\
\hline \multicolumn{5}{|c|}{ ^ Lower scores indicate better performances } \\
\hline $\begin{array}{l}\text { Abbreviations: } \mathrm{RT}=\text { reaction } \\
\mathrm{CG}=\text { Control Group. }\end{array}$ & ;SSPA = Sit-sta & desks and PA & ded group; PA & group; \\
\hline
\end{tabular}


Table 4

Baseline characteristics of sleep habits among the study participants

$\begin{array}{lll}\text { SSPA } & \text { PA } & \text { CG } \\ (n=23) & (n=25) & (n=28)\end{array}$

\section{Sleep habits}

Usual night wakeups

5-7 times/week (\%)

8.7

8.0

7.1

1-4 times/week (\%)

17.4

16.0

10.7

2-3 times/month (\%)

43.5

28.0

28.6

Never (\%)

30.4

48.0

53.6

Back to sleep latency after wakeups

Never wake up (\%)

36.4

44.0

42.9

Very quickly (\%)

18.2

12.0

14.3

5-10 $\min (\%)$

31.8

32.0

25.0

$10-30 \min (\%)$

4.5

8.0

14.3

More than $30 \mathrm{~min}(\%)$

9.1

4.0

3.6

Nap frequency

Never (\%)

39.1

36.0

35.7

Never unless sick (\%)

47.8

36.0

39.3

Sometimes (\%)

13.0

24.0

17.9

Almost every day (\%)

0

4.0

7.1

Perceived sleep duration

Too much (\%)

13.0

4.0

3.6

Right amount (\%)

60.9

80.0

75.0

Too little (\%)

26.1

16.0

21.4

Perceived sleep quality

Great (\%)

13.0

20.0

35.7

Good (\%)

56.5

48.0

46.4

Abbreviations: SSPA = Sit-stand desks and PA blended group; $\mathrm{PA}=$ single PA group; $\mathrm{CG}=$ Control Group 


\begin{tabular}{|llll|}
\hline & $\begin{array}{l}\text { SSPA } \\
(\mathbf{n = 2 3})\end{array}$ & $\begin{array}{l}\text { PA } \\
(\mathbf{n = 2 5})\end{array}$ & $\begin{array}{l}\text { CG } \\
(\mathbf{n}=\mathbf{2 8})\end{array}$ \\
\hline Okay (\%) & 26.1 & 28.0 & 14.3 \\
\hline Poor (\%) & 4.3 & 4.0 & 3.6 \\
\hline $\begin{array}{l}\text { Abbreviations: SSPA } \\
\text { Group }\end{array}$ & Sit-stand desks and PA blended group; PA & single PA group; CG = Control \\
\hline
\end{tabular}

\section{Discussion}

The current study pioneers to incorporate sit-stand desks and PA breaks as the active strategy to not only reduce students' sitting time and increase their PA engagement, but also investigate the benefits of this intervention on children's PL, various patterns in sleep, and executive functions. This could provide a multi-level appraisal to assist students in pursuing healthy lifestyle among Hong Kong primary schools. As children spend the majority of their waking time being sedentary at school, environmental changes in the classroom could be an effective way of reducing their sitting time (10). Although sit-stand desk interventions have been employed in many western countries to promote children's health, previous studies were restricted to only reducing or disrupting prolonged sitting time. Thus, to provide an active environment for children, such as combining PA classroom breaks with sit-stand desks, would be a better approach for increasing their engagement in PA, especially MVPA, as public health authorities have made a recommendation to primary schools to help children meet their $60 \mathrm{~min} /$ day PA guideline (65).

The baseline characteristics showed that it is satisfactory to conduct the current research design, which may contribute to the incorporation of sit-stand desks and PA breaks into the blended "Stand + Move" intervention and optimize a blended approach to increase children's engagement in PA and PL development. Meanwhile, as the baseline results are satisfactory, it could be better to enhance children's executive functioning and a co-development of PA, SB and sleep within 24-hour for students in Hong Kong primary schools.

Although traditional classrooms and educational institutions in Hong Kong encourage adopting innovative teaching methods such as incorporating fundamental movement skills into PE class content, this is the first attempt at a research-based intervention combining children's learning environment with healthy lifestyle nurturing. Many countries who rank within the top 25 for obesity prevalence have emphasized the importance of the PL concept as a guiding ideology in their policies and programs in response to both global PA decline and SB accumulation (66). With its focus on encompassing "motivation, confidence, physical competence, and knowledge and understanding," to value and take responsibility for engagement in PA for life (67), PL would work as a guiding ideology for conceptualizing the current research and providing scope for embodied enrichment on the pathway toward an active lifestyle. 
This experimental research serves as a pioneering study to combine sit-stand desks with PA active breaks in Hong Kong's primary school environment. The RCT's aim is to investigate the effects of a classroombased teacher-led intervention on children's PL, PA, sleep, and executive functions. The present study is significant and innovative in that it reconstructs an active classroom environment for students in primary schools to promote a healthy lifestyle; physical fitness and motor skills; executive functions; and enhances the healthy pattern of sleep through comprehensive objective and subjective measures. As the 24-Hour Movement Guideline for Children and Youth emphasizes PA, SB, and sleep as three codevelopmental movement behaviors relating to the full scope of movement within a 24-h period (13). To the best of our knowledge, no previous investigations have combined PL, PA, and sleep changes using environmentally-driven strategies, while simultaneously including comprehensive measures within different fields. The current study also outlines the evidence-based, health-related benefits of the "Stand + Move" intervention for expanded use with Hong Kong children, and promotes sit-stand desks and active classroom strategies for more primary schools. In addition, it emphasizes the concept of PL, which has been adopted as the new standard to evaluate PE outcomes in many other countries, when implementing PA interventions for children in Hong Kong. This study may demonstrate the longitudinal benefit of incorporating sit-stand desks to improve students' executive function and academic achievements.

However, the baseline results of this study has shown some insights for limitations. First, as a full desk allocation system (a sit-stand desk for every child) guaranteed the optimal health benefits for the participants, only one school was recruited for this study. Therefore, the present intervention should be interpreted with some caution regarding its generalizability of the trial findings. Second, when adopting activPAL for use in school-aged children, a great drop-out rate occurred during the wear period even though several strategies were applied to avoid unnecessary removal, such as using the alcohol pads to clean the skin before attachment, and put cartoon stickers to attract children's attention according to the previous suggestions (68). This is consistent with previous studies conducted in preschool children (69) and primary students (68) because skin irritation problems such as itchy skin and allergic reactions were more prevalent in the wet environment, especially in Hong Kong with such a humid weather. The present study may not be able to provide sufficient evidence for activPAL data in the following measurements.

Nonetheless, with regard to the high prevalence and harmful effects of sedentary lifestyles in children and youth, effective interventions to motivate participation in PA in different contexts are warranted. Considering this study's valuable and detailed research design, we hypothesize that its "Stand + Move" RCT will be effective in increasing several aspects of children's PL, PA, sleep, and executive functions. The study's direct beneficiaries are expected to be:

Children in Hong Kong primary schools participating in the "Stand + Move" intervention. They will enhance their PL and co-develop their PA, SB, and sleep, as well as their executive functions and academic performance. In addition, they will benefit from receiving a report on their performances in both the physical and psychosocial assessments. Although children in the control group were not involved in the sit-stand desk or PA break interventions, this intervention will enhance their understanding of their own performances, in both the physical and psychological assessments (short and long term).

Page $18 / 26$ 
All stakeholders of the participants will benefit since they will have access to resources and relevant information related to the "Stand + Move" intervention, as well as the rationale behind it. More participation time in PA and less time spent in SB will inspire a more active lifestyle in their PL journey. As a result of participating in this project, the stakeholders-principals, teachers, parents, and guardians-will also benefit through enhancements in their children's health-related physical and psychological development. Furthermore, enhancing children's academic achievements will provide the desired outcomes to meet parents' expectations. Finally, this collaborative project will create awareness amongst practitioners in Hong Kong e.g., Non-Profit Organizations, Hong Kong Physical Fitness Association, etc., to promote such feasible and innovative classroom environments for pursuing healthy living through more effective engagement, for both the short and long term).

Policymakers in government departments such as Hong Kong's Education Bureau, Education Commission, and Quality Education Department will benefit from the research results which will provide evidence to support their policy-related decisions in relation to reducing and disrupting SB, thus promoting PA throughout a child's life. Furthermore, they will benefit from collaborating with university research centers and school practitioners to provide sustainable and quality strategies and services to improve children's health behaviors, which in return, will decrease the government's childcare expenditures. The public will also have an enhanced understanding of the efficiency and effectiveness of the "Stand + Move" program to reduce sitting time, and promote participation in PA. At the macro-level, this study may inform relevant Hong Kong government officials about the importance of reconstructing classroom environments, so as to create a space where children's healthy lifestyle habits may be nurtured through the promotion of sit-stand desks and PA breaks

Hong Kong Physical Fitness Association will benefit from providing professional training to PE practitioners evaluating children's PL. A certificate named "Childhood Physical Literacy Leader Certificate" will be promoted among PE practitioners in Hong Kong to enhance their competence in the field of education.

\section{Conclusions}

The baseline results suggest that this study was well designed and implemented and has been satisfactory so far. This blended classroom-based intervention is expected to provide empirical evidence of enhancements in school-aged children's PL, PA, executive function and sleep, with a special focus on fostering them to meet more movement guidelines within 24-hour period.

\section{Abbreviations}

CAMSA

the Canadian agility and movement skill assessment CSPAP Comprehensive School Physical Activity Program 
MVPA

Moderate-to-vigorous physical activity

PA

Physical activity

PACER

Progressive Aerobic Cardiovascular Endurance Run

PE

Physical education

PL

Physical literacy

SB

Sedentary behavior

\section{Declarations}

\section{Ethics approval and consent to participate}

The protocol of the study was reviewed and approved by Survey and Behavioral Research Ethics of the Chinese University of Hong Kong (No: SBRE-18-108). Written informed consent will be obtained from participating students and parents prior to the start of the trial.

\section{Funding}

This work was funded by the Direct Grant for Research (Grant No. EDU 2019-052) of the Chinese University of Hong Kong. The funding body did not take part in the design of the study, the collection, analysis, and interpretation of data, and preparation of the manuscript.

\section{Author Contributions}

RKWS, CHPS and MHL conceived the study, YKL provided professional suggestions in children's sleep measures. All authors were involved in the design of the study protocol and assisted with the drafting and revising of the manuscript, read and approved the final manuscript.

\section{Acknowledgments}

We would like to thank Principal Ng from Pooi Kei Primary School, Mr. Sam Wong and Dr. Jacky Chan from Hong Kong Physical Fitness Association, and all the involved teachers and primary school students for all their support with data collection procedures.

\section{Consent for publication}

Not applicable.

\section{Competing Interests}


The authors declare that they have no competing interests.

\section{Availability of data and materials}

Due to data protection regulations, there is no permission to make the datasets available to third parties.

\section{References}

1. Ridgers ND, Timperio A, Cerin E, Salmon J. Within- and between-day associations between children's sitting and physical activity time. Bmc Public Health. 2015;15.

2. Huang WY, Wong SHS, Sit CHP, Wong MCS, Sum RKW, Wong SWS, et al. Results from the Hong Kong's 2018 report card on physical activity for children and youth. J Exerc Sci Fit. 2019;17(1):14-9.

3. Huang WY, Wong SH, He G, Salmon JO. Isotemporal Substitution Analysis for Sedentary Behavior and Body Mass Index. Med Sci Sports Exerc. 2016;48(11):2135-41.

4. Clemes SA, Barber SE, Bingham DD, Ridgers ND, Fletcher E, Pearson N, et al. Reducing children's classroom sitting time using sit-to-stand desks: findings from pilot studies in UK and Australian primary schools. J Public Health (Oxf). 2016;38(3):526-33.

5. Tremblay MS, LeBlanc AG, Kho ME, Saunders TJ, Larouche R, Colley RC, et al. Systematic review of sedentary behaviour and health indicators in school-aged children and youth. Int J Behav Nutr Phys Act. $2011 ; 8: 98$.

6. Marshall SJ, Biddle SJH, Gorely T, Cameron N, Murdey I. Relationships between media use, body fatness and physical activity in children and youth: a meta-analysis. Int J Obesity. 2004;28(10):123846.

7. Biddle SJ, Pearson N, Ross GM, Braithwaite R. Tracking of sedentary behaviours of young people: a systematic review. Prev Med. 2010;51(5):345-51.

8. Tomporowski PD, Lambourne K, Okumura MS. Physical activity interventions and children's mental function: an introduction and overview. Prev Med. 2011;52 Suppl 1:S3-9.

9. Carlson JA, Engelberg JK, Cain KL, Conway TL, Mignano AM, Bonilla EA, et al. Implementing classroom physical activity breaks: Associations with student physical activity and classroom behavior. Preventive Medicine. 2015;81:67-72.

10. Castelli DM, Centeio EE, Beighle AE, Carson RL, Nicksic HM. Physical literacy and Comprehensive School Physical Activity Programs. Prev Med. 2014;66:95-100.

11. Whitehead M. Physical Literacy Throughout the Lifecourse. London: Routledge Taylor \& Francis Group; 2010.

12. Education Bureau. An overview of the learning topics in the six strands: Physical education key learning area. In: Department GL, editor. Bureau E. Hong Kong2015.

13. Carson V, Ezeugwu VE, Tamana SK, Chikuma J, Lefebvre DL, Azad MB, et al. Associations between meeting the Canadian 24-Hour Movement Guidelines for the Early Years and behavioral and 
emotional problems among 3-year-olds. J Sci Med Sport / Sports Medicine Australia. 2019;22(7):797-802.

14. Cairney J, Dudley D, Kwan M, Bulten R, Kriellaars D. Physical Literacy, Physical Activity and Health: Toward an Evidence-Informed Conceptual Model. Sports Med. 2019;49(3):371-83.

15. Salmon J, Koorts H, Timperio A. Specific Interventions Targeting Sedentary Behaviour in Children and Adolescents. Springer Ser Epidemi. 2018:431-43.

16. De Meester F, Van Dyck D, De Bourdeaudhuij I, Deforche B, Cardon G. Changes in physical activity during the transition from primary to secondary school in Belgian children: what is the role of the school environment? Bmc Public Health. 2014;14.

17. Soderlund PD. The Social Ecological Model and Physical Activity Interventions for Hispanic Women With Type 2 Diabetes: A Review. J Transcult Nurs. 2017;28(3):306-14.

18. Naylor PJ, McKay HA. Prevention in the first place: schools a setting for action on physical inactivity. Br J Sports Med. 2009;43(1):10-3.

19. Murtagh E, Mulvihill M, Markey O. Bizzy Break! The effect of a classroom-based activity break on inschool physical activity levels of primary school children. Pediatr Exerc Sci. 2013;25(2):300-7.

20. Carson V, Lee EY, Hewitt L, Jennings C, Hunter S, Kuzik N, et al. Systematic review of the relationships between physical activity and health indicators in the early years (0-4 years). Bmc Public Health. 2017;17.

21. Best JR, Miller PH. A Developmental Perspective on Executive Function. Child Dev. 2010;81(6):164160.

22. Bezerra TA, Clark CCT, De Souza AN, Fortes LD, Mota JAPS, Duncan MJ, et al. 24 movement behaviour and executive function in preschoolers: A compositional and isotemporal reallocation analysis. European journal of sport science. 2020.

23. Turnbull K, Reid GJ, Morton JB. Behavioral Sleep Problems and their Potential Impact on Developing Executive Function in Children. Sleep. 2013;36(7):1077-84.

24. Crova C, Struzzolino I, Marchetti R, Masci I, Vannozzi G, Forte R, et al. Cognitively challenging physical activity benefits executive function in overweight children. Journal of sports sciences. 2014;32(3):201-11.

25. Lillard AS, Peterson J. The Immediate Impact of Different Types of Television on Young Children's Executive Function. Pediatrics. 2011;128(4):644-9.

26. Walsh JJ, Barnes JD, Cameron JD, Goldfield GS, Chaput JP, Gunnell KE, et al. Associations between 24 hour movement behaviours and global cognition in US children: a cross-sectional observational study. Lancet Child Adolesc. 2018;2(11):783-91.

27. Chaput JP, Carson V, Gray CE, Tremblay MS. Importance of All Movement Behaviors in a 24 Hour Period for Overall Health. Int J Env Res Pub He. 2014;11(12):12575-81.

28. Mazzoli E, Teo WP, Salmon J, Pesce C, He J, Ben-Soussan TD, et al. Associations of Class-Time Sitting, Stepping and Sit-to-Stand Transitions with Cognitive Functions and Brain Activity in Children. 
Int J Environ Res Public Health. 2019;16(9).

29. Jager K, Schmidt M, Conzelmann A, Roebers CM. The effects of qualitatively different acute physical activity interventions in real-world settings on executive functions in preadolescent children. Ment Health Phys Act. 2015;9:1-9.

30. Hinckson E, Salmon J, Benden M, Clemes SA, Sudholz B, Barber SE, et al. Standing Classrooms: Research and Lessons Learned from Around the World. Sports Med. 2016;46(7):977-87.

31. Salmon J. Novel Strategies to Promote Children's Physical Activities and Reduce Sedentary Behavior. J Phys Act Health. 2010;7:S299-S306.

32. Pearson N, Haycraft E, Johnston JP, Atkin AJ. Sedentary behaviour across the primary-secondary school transition: A systematic review. Preventive Medicine. 2017;94:40-7.

33. Campbell MK, Piaggio G, Elbourne DR, Altman DG, Grp C. Consort 2010 statement: extension to cluster randomised trials. Bmj-Brit Med J. 2012;345.

34. Edwards LC, Bryant AS, Keegan RJ, Morgan K, Cooper SM, Jones AM. 'Measuring' Physical Literacy and Related Constructs: A Systematic Review of Empirical Findings. Sports Med. 2018;48(3):659-82.

35. Li MH, Sum RKW, Sit CHP, Wong SHS, Ha ASC. Associations between perceived and actual physical literacy level in Chinese primary school children. Bmc Public Health. 2020;20(1).

36. Faul F, Erdfelder E, Lang AG, Buchner A. G*Power 3: A flexible statistical power analysis program for the social, behavioral, and biomedical sciences. Behav Res Methods. 2007;39(2):175-91.

37. Li MH, Sum RKW, Tremblay M, Sit CHP, Ha ASC, Wong SHS. Cross-validation of the Canadian Assessment of Physical Literacy second edition (CAPL-2): The case of a Chinese population. Journal of sports sciences. 2020:1-8.

38. Longmuir PE. Understanding the Physical Literacy Journey of Children: the Canadian Assessment of Physical Literacy. Bulletin - Journal of Sport Science and Physical Educational Theory. 2013;65:27682.

39. Meredith MD, Welk GJ. FitnessGram \& ActivityGram: Test Administration Manual. Dallas, Texas: The Cooper Institute; 2010.

40. Boyer C, Tremblay M, Saunders T, McFarlane A, Borghese M, Lloyd M, et al. Feasibility, Validity, and Reliability of the Plank Isometric Hold as a Field-Based Assessment of Torso Muscular Endurance for Children 8-12 Years of Age. Pediatric Exercise Science. 2013;25(3):407-22.

41. Longmuir PE, Boyer C, Lloyd M, Borghese MM, Knight E, Saunders TJ, et al. Canadian Agility and Movement Skill Assessment (CAMSA): Validity, objectivity, and reliability evidence for children 8-12 years of age. Journal of Sport and Health Science. 2017;6(2):231-40.

42. Hay JA. Adequacy in and predilection for physical activity in children. Clinical Journal of Sport Medicine. 1992;2(3):192-201.

43. Longmuir PE, Gunnell KE, Barnes JD, Belanger K, Leduc G, Woodruff SJ, et al. Canadian Assessment of Physical Literacy Second Edition: a streamlined assessment of the capacity for physical activity among children 8 to 12 years of age. Bmc Public Health. 2018;18. 
44. Sum RKW, Cheng CF, Wallhead T, Kuo CC, Wang FJ, Choi SM. Perceived physical literacy instrument for adolescents: A further validation of PPLI. Journal of Exercise Science \& Fitness. 2018;16(1):2631.

45. Sum RKW, Ha AS, Cheng CF, Chung PK, Yiu KT, Kuo CC, et al. Construction and Validation of a Perceived Physical Literacy Instrument for Physical Education Teachers. Plos One. 2016;11(5):e0155610.

46. Robusto KM, Trost SG. Comparison of three generations of ActiGraph (TM) activity monitors in children and adolescents. Journal of sports sciences. 2012;30(13):1429-35.

47. Evenson KR, Catellier DJ, Gill K, Ondrak KS, McMurray RG. Calibration of two objective measures of physical activity for children. Journal of sports sciences. 2008;26(14):1557-65.

48. Herrmann SD, Barreira TV, Kang M, Ainsworth BE. How Many Hours Are Enough? Accelerometer Wear Time May Provide Bias in Daily Activity Estimates. J Phys Act Health. 2013;10(5):742-9.

49. Kozey-Keadle S, Libertine A, Lyden K, Staudenmayer J, Freedson PS. Validation of Wearable Monitors for Assessing Sedentary Behavior. Med Sci Sport Exer. 2011;43(8):1561-7.

50. Shi Y, Huang WY, Yu JJ, Wong SHS. Compliance With The 24-h Movement Guidelines In Hong Kong Adolescents: Associations With Body Mass Index. Med Sci Sport Exer. 2019;51(6):521-.

51. Dowd KP, Harrington DM, Bourke AK, Nelson J, Donnelly AE. The measurement of sedentary patterns and behaviors using the activPAL (TM) Professional physical activity monitor. Physiol Meas. 2012;33(11):1887-99.

52. Eriksen BA, Eriksen CW. Effects of noise letters upon the identification of a target letter in a nonsearch task. Percept Psychophysiology. 1974;16:143-9.

53. Heaton RK, Chelune GJ, Talley JL, Kay GG, Curtiss G. Wisconsin card sorting test manual: revised and expanded. Odessa, FL1993.

54. Del Giorno JM, Hall EE, O'Leary KC, Bixby WR, Miller PC. Cognitive Function During Acute Exercise: A Test of the Transient Hypofrontality Theory. Journal of Sport \& Exercise Psychology. 2010;32(3):31223.

55. Anderson P, Anderson V, Lajoie G. The Tower of London Test: Validation and standardization for pediatric populations. Clin Neuropsychol. 1996;10(1):54-65.

56. Chang YK, Tsai CL, Hung TM, So EC, Chen FT, Etnier JL. Effects of Acute Exercise on Executive Function: A Study With a Tower of London Task. Journal of Sport \& Exercise Psychology. 2011;33(6):847-65.

57. Meltzer LJ, Avis KT, Biggs S, Reynolds AC, Crabtree VM, Bevans KB. The Children's Report of Sleep Patterns (CRSP): a self-report measure of sleep for school-aged children. J Clin Sleep Med. 2013;9(3):235-45.

58. Drake C, Nickel C, Burduvali E, Roth T, Jefferson C, Badia P. The pediatric daytime sleepiness scale (PDSS): Sleep habits and school outcomes in middle-school children. Sleep. 2003;26(4):455-8. 
59. Patton MQ. Qualitative research \& evaluation methods : integrating theory and practice. Fourth edition. ed. Thousand Oaks, California: SAGE Publications, Inc.; 2015. xxi, 806 pages $\mathrm{p}$.

60. Moore GF, Audrey S, Barker M, Bond L, Bonell C, Hardeman W, et al. Process evaluation of complex interventions: Medical Research Council guidance. BMJ. 2015;350:h1258.

61. McKenzie G, Powell J, Usher R. Understanding Social Research: Perspectives on Methodology and Practice. London: Falmer Press; 1997.

62. Hammersley M, Atkinson P. Recording and organizing data. Ethnography: Principles in Practice. London: Routledge; 1995.

63. Kang M, Rowe DA, Barreira TV, Robinson TS, Mahar MT. Individual Information-Centered Approach for Handling Physical Activity Missing Data. Research quarterly for exercise and sport. 2009;80(2):131-7.

64. Tremblay MS, Carson V, Chaput JP, Gorber SC, Dinh T, Duggan M, et al. Canadian 24-Hour Movement Guidelines for Children and Youth: An Integration of Physical Activity, Sedentary Behaviour, and Sleep. Appl Physiol Nutr Me. 2016;41(6):S311-S27.

65. Strong WB, Malina RM, Blimkie CJR, Daniels SR, Dishman RK, Gutin B, et al. Evidence based physical activity for school-age youth. J Pediatr-Us. 2005;146(6):732-7.

66. Spengler J. Physical literacy global environmental scan. 2014.

67. International Physical Literacy Association. The definition of physical literacy UK2017. Available from: https://www.physical-literacy.org.uk/.

68. Shi Y, Huang WYJ, Yu JJ, Sheridan S, Sit CHP, Wong SHS. Compliance and Practical Utility of Continuous Wearing of activPAL (TM) in Adolescents. Pediatric Exercise Science. 2019;31(3):363-9.

69. De Decker E, De Craemer M, Santos-Lozano A, Van Cauwenberghe E, De Bourdeaudhuij I, Cardon G. Validity of the ActivPAL (TM) and the ActiGraph Monitors in Preschoolers. Med Sci Sport Exer. 2013;45(10):2002-11.

\section{Figures}




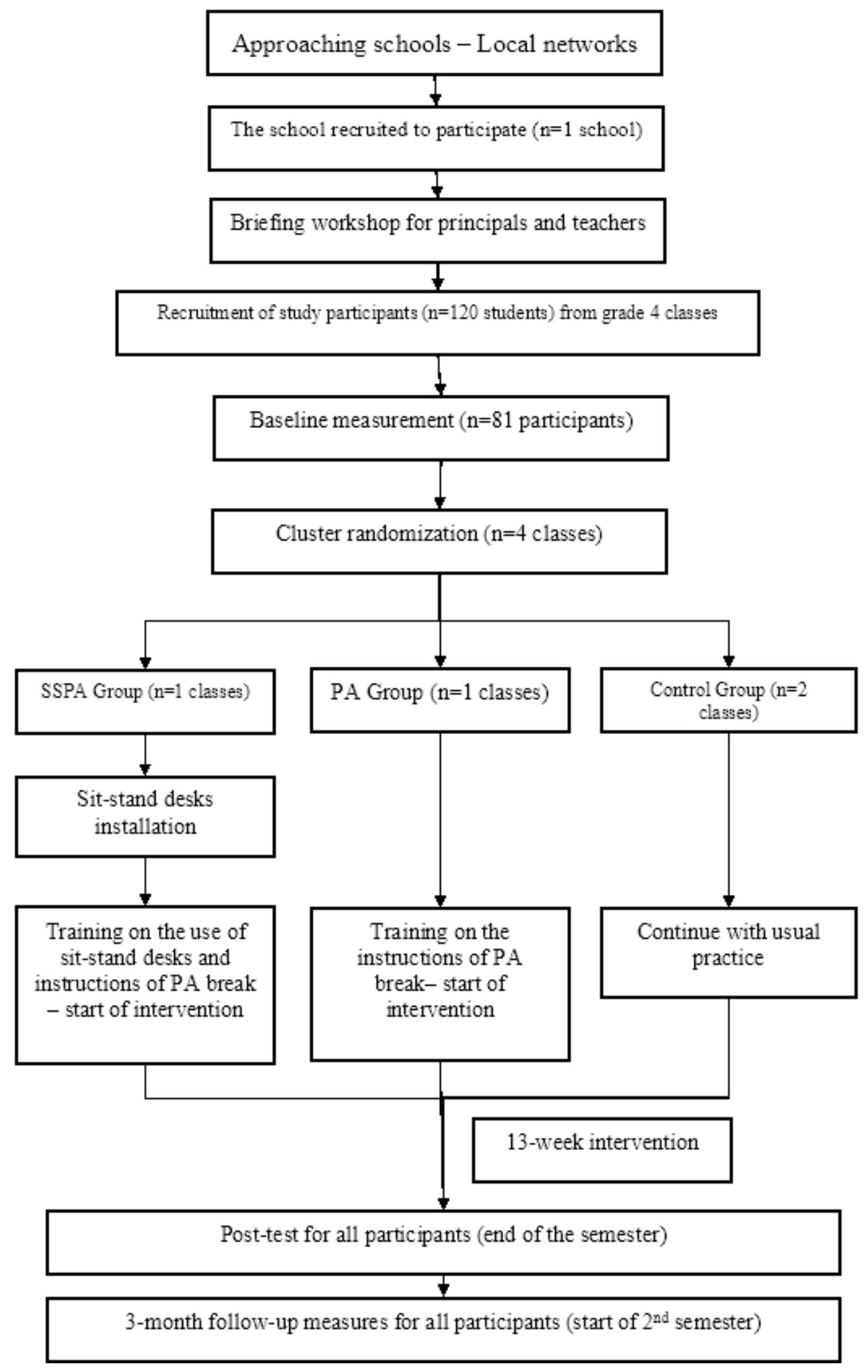

\section{Figure 1}

Flow diagram for the "Stand+ Move" intervention using CONSORT guidelines for RCT 